\title{
Surface Morphology and Molecular Arrangement of Organized Films of Poly-(N-alkylated benzamides) Containing Various Chain Lengths
}

\author{
Natski Sato ${ }^{1}$, Satoshi Chiba ${ }^{2},{\text { Yoko } \mathrm{Abe}^{3} \text {, Yuji Shibasaki }}^{3}$, and Atsuhiro Fujimori ${ }^{1 *}$ \\ ${ }^{1}$ Graduate School of Science and Engineering, Yamagata University, Jonan 4-3-16, Yonezawa, Yamagata, 992-8510, Japan \\ ${ }^{2}$ Department of Polymer Science and Engineering, Yamagata University, Jonan 4-3-16, Yonezawa, Yamagata, 992-8510, Japan \\ ${ }^{3}$ Department of Chemistry and Bioengineering, Iwate University, Ueda 4-3-5, Morioka, Iwate, 020-8551, Japan.
}

Fax: +81-238-26-3073, e-mail: fujimori@yz.yamagata-u.ac.jp

\begin{abstract}
We investigated the molecular arrangement and surface morphology of organized molecular films with regard to solid-state structures for newly synthesized aromatic polyamides with various side chain length by surface pressure-area $(\pi-\mathrm{A})$ isotherm, in-plane and out-of plane X-ray diffraction (XRD), and atomic force microscopy $(\mathrm{AFM})$. Since the aromatic polyamides have rigid main chain and flexible side chain, it may be possible to form the phase separated structure at sub-nanometer scales. These polyamides form the extremely condensed monolayer on the water surface. Multilayers of these polyamides transferred by Langmuir-Blodgett (LB) method construct highly order layer structure. On the other hand, in the two-dimensional film plane of polyamides, amorphous structure of side chains is formed except for octadecyl derivatives. Changes in the surface morphology of monolayers for these polyamides definitely depend on the side chain length. Spherulitic, fibril like, and homogeneous flat morphologies of the film surface are systematically observed by AFM measurement.
\end{abstract}

Key words: Aromatic Polyamides, Langmuir-Blodgett Film, Monolayers, Surface Morphology, Molecular arrangement

\section{INTRODUCTION}

Functional polymers, which have precisely controlled molecular arrangements, and their organized molecular films ${ }^{1}$ can be developed as potential candidates for fabricating biomimetic models ${ }^{2}$ and molecular electronic devices, ${ }^{3}$ which have attracted considerable interest in fundamental science; further, these molecular assemblies have several other potential applications. $^{4}$

It is commonly known that crystalline polymers can be used to form hierarchical structures ranging in size from nanometer-scale lamellae to mesoscopic-scale spherulites. ${ }^{5}$ These crystalline polymers are generally formed by the folding of the main chain. Side chain crystalline comb polymers show two types of structural characteristics. It is known that comb polymers, which have crystallizable long n-alkyl side chains attached to an amorphous main chain, are packed into a layer structure along the $c$-axis and a subcell consisting of the side chains in the $a b$-plane. ${ }^{6} \quad$ This subcell structure ${ }^{7}$ is formed as a two-dimensional lattice, and the layer structure along the $c$-axis often reflects the large spacing between the main chains in the accumulated double layer structures. Octadecyl-based materials, which are types of long-chain compounds and are co-crystallized with another hydrocarbon to incorporate the hydrocarbon into the same crystalline lattice, have found numerous industrial applications such as pour point depressants for lubricating oils or fuels, rheological modifiers, additives for petroleum products, and smart gels. ${ }^{8}$

Plate and Shibaev ${ }^{9}$ have studied these comb polymers extensively. An X-ray diffraction profile of the comb polymers showed an intense reflection corresponding to a Bragg spacing $d$ of 4.2 $\AA$, which suggested the formation of a hexagonal crystalline lattice of n-alkyl side chains. Further, reflection occurred in small-angle regions, which was considered to originate from the layer structure. The periodicity of the crystalline-amorphous layer can be obtained by estimating the diffraction angle of this reflection.

In the present study, new aromatic polyamides with a rigid main chain and flexible side chain having different lengths (poly-(N-alkylated benzamide), abbrev. $\mathrm{PABA}_{\mathrm{n}}$, Fig. 1) were synthesized. The solid-state structures, i.e., the molecular orientation and surface morphology, of organized molecular films of these polymers were investigated by performing surface pressure-area $(\pi-\mathrm{A})$ isotherm, in-plane and out-of plane X-ray diffraction (XRD), and atomic force microscopy (AFM) measurements. It was possible to control the molecular arrangement and morphologies of these systematic polymer samples by preparing organized molecular films. Further, we have 
(a)

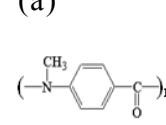

(b)

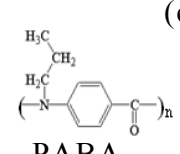

(c)

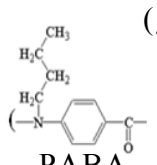

$\mathrm{PABA}_{1}$

(d)

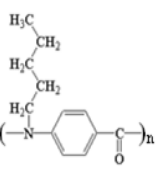

$\mathrm{PABA}_{5}$ (e) Н $_{\text {c }}$

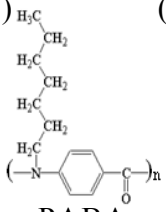

$\mathrm{PABA}_{7}$ (g) ${ }^{\mathrm{H}}$

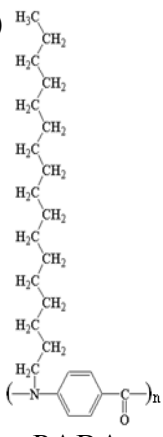

$\mathrm{PABA}_{17}$

Figure 1 Chemical structure of aromatic polyamides with various side chains: (a) methyl, (b) propyl, (c) butyl, (d) pentyl, (e) heptyl, (f) octyl, and (g) stearyl substituents.

discussed the possibility of using these polyamides as new polymer nanomaterials.

\section{EXPERIMENTAL}

2.1 Materials $^{10}$

2.1.1 Direct polycondensation of PABA ${ }_{n}$.

$\mathrm{PABA}_{\mathrm{n}}$ compounds used in this study were synthesized by the direct condensation polymerization of $\mathrm{N}$-alkylated aminobenzoic acid with methyl, propyl, butyl, pentyl, heptyl, octyl, and stearyl substituents. $\mathrm{PABA}_{1}$ was prepared and poured into a three-necked flask equipped with a reflux condenser, a three-way stopcock, and a thermometer. N-Methylaminobeozoic acid $(4.55 \mathrm{~g}, 30.1 \mathrm{~mol})$, triphenylphosphine $(9.44 \mathrm{~g}$, $36.0 \mathrm{mmol})$, and pyridine $(30 \mathrm{~mL})$ were then added to the flask. The mixture was stirred until the solid was dissolved. Hexachloroethane $(8.52 \mathrm{~g}, 36.0 \mathrm{mmol})$ was then added to this solution, and the mixture was refluxed for $24 \mathrm{~h}$. After the solution was cooled to room temperature, methanol/hydrogen chloride was added and stirred for $5 \mathrm{~h}$. The precipitate was collected, washed with hot water, and dried at $220{ }^{\circ} \mathrm{C}$ for $24 \mathrm{~h}$ to give the $\mathrm{PABA}_{1}$ compound. The other $\mathrm{PABA}_{\mathrm{n}}$ compounds were prepared by the same method, as described above. The average molecular weights, $M_{\mathrm{w}}$, of these aromatic polyamides were $20,000-90,000$.

\subsection{Formation of polymer monolayers on water} surface and observation of molecular arrangement in the films.

Monolayers of aromatic polyamides prepared from $\mathrm{m}$-cresol or chloroform or trifluoroacetic acid solutions (about $10^{-4} \mathrm{M}$ ) were formed on distilled water (about $18 \mathrm{M} \Omega \mathrm{cm}$ ). The surface pressure-area $(\pi-\mathrm{A})$ isotherms of the polyamide monolayers were measured on a film balance (Kyowa Kaimen Kagaku Co. Ltd., compression speed: $3 \mathrm{~cm} / \mathrm{min}$ ) at $15{ }^{\circ} \mathrm{C}$. These aromatic polyamides formed highly condensed monolayers. These monolayers were transferred onto solid (glass) substrates at $15{ }^{\circ} \mathrm{C}$, and an appropriate surface pressure $\left(15 \sim 25 \mathrm{mNm}^{-1}\right)$ was applied to obtain alternating Y-type films by the

Langmuir-Blodgett (LB) method. ${ }^{1}$ Hydrophobic side chains on the outermost surface of Z-type LB films transferred onto solid substrates were exposed to air.

The surface morphologies of the transferred films were observed using a scanning probe microscope (Seiko Instrument, SPA300 with SPI-3800 probe station) and microfabricated rectangular $\mathrm{Si}$ cantilevers with integrated pyramidal tips by applying a constant force of 1.4 $\mathrm{N} / \mathrm{m}$. The large spacing between the layer structures of the films transferred onto the glass substrates were measured using an out-of plane $\mathrm{X}$-ray diffractometer (Rigaku, Rad-rA, $\mathrm{CuK \alpha}$ radiation, $40 \mathrm{kV}, 100 \mathrm{~mA}$ ) equipped with a graphite monochromator. The in-plane spacing of the two-dimensional lattice of the films was determined using an X-ray diffractometer with different geometrical arrangements ${ }^{11}$ (Bruker AXS, MXP-BX, $\mathrm{CuK \alpha}$ radiation, $40 \mathrm{kV}, 40 \mathrm{~mA}$, an instrument specially made to order) and equipped with a parabolic graded multilayer mirror. The X-rays were incident at an angle of $0.2^{\circ}$, and the films were slow scanned at a speed of $0.05^{\circ} / 20 \mathrm{~s}$; thus, in-plane XRD measurements were carried out at a monomolecular resolution.

\section{RESULTS and DISCUSSION}

3.1 Molecular arrangement of organized molecular films of aromatic polyamides.

From the results of $\pi-\mathrm{A}$ isotherm at $15{ }^{\circ} \mathrm{C}$, extremely condensed monolayers of aromatic polyamides formed on the water surface, the presence of two condensed states and plateau regions is confirmed. Out-of plane XRD profiles of the poly-(N-alkylated benzamide) LB multilayers transferred at the second condensed phase formed on the water surface are shown in Fig. 2. Quite interestingly, all the polymers
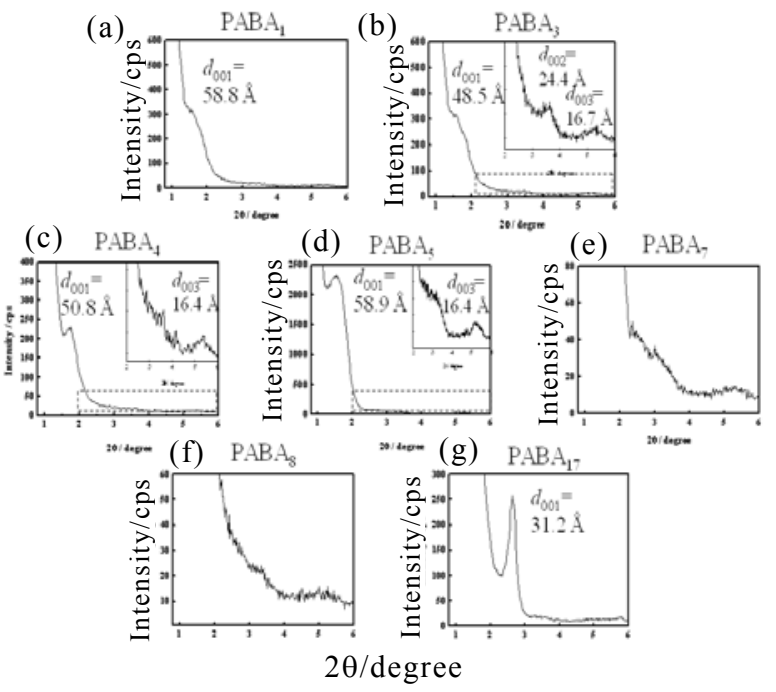

Figure 2 Out-of plane XRD profiles of LB multilayers $\left(2 \theta=2 \sim 6^{\circ}, 20\right.$ layers $)$ of aromatic polyamides with various side chains: (a) methyl, (b) propyl, (c) butyl, (d) pentyl, (e) heptyl, (f) octyl, and (g) stearyl substituents. 
(a)
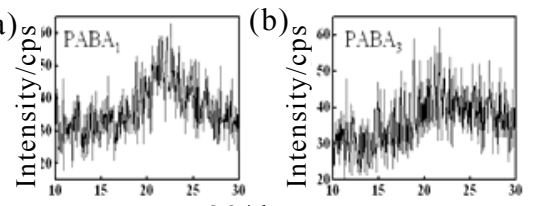
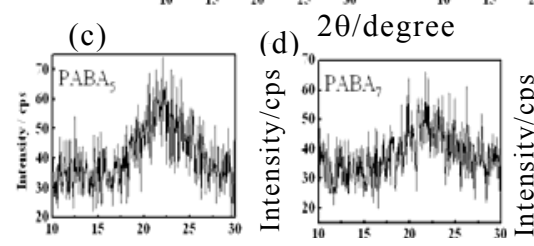

$2 \theta /$ degree
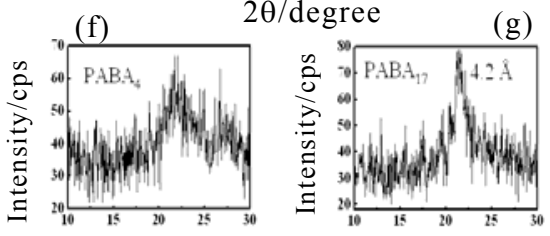

$2 \theta /$ degree

Figure 3 In-plane XRD profiles of LB multilayers (20 layers) of aromatic polyamides with various side chains: (a) methyl, (b) propyl, (c) butyl, (d) pentyl, (e) heptyl, (f) octyl, and (g) stearyl substituents.

except $\mathrm{PABA}_{7}$ and $\mathrm{PABA}_{8}$ show a layer spacing of around $50-60 \AA$ along the $c$-axis. Only the PABA $_{17}$ LB film shows almost the same large spacing as that in the bulk state. The LB films of $\mathrm{PABA}_{1-8}$ show very large spacing. On the other hand, in-plane XRD measurements indicate that these LB films show irregularity in the film plane (Fig. 3). Only the $\mathrm{PABA}_{17}$ multilayer shows a two-dimensional lattice spacing of $4.2 \AA$ due to the formation of a subcell structure made of the stearyl side chain. All the other polyamide LB multilayers form an amorphous structure in the $a b$-plane. In general, amorphous polymer LB films with a nanometer-order stable layered structure are highly durable. ${ }^{12}$ These aromatic polyamide LB films can be used to fabricate new nanomaterials because of their amorphous layered structure.

3.2 Side chain length dependency of surface morphology of aromatic polyamide monolayers.

Figure 4 shows AFM images of Z-type aromatic polyamide monolayers transferred onto a mica substrate. Observations suggest that the $\mathrm{PABA}_{1}$, $\mathrm{PABA}_{3}, \mathrm{PABA}_{4}$, and $\mathrm{PABA}_{5}$ films are single particle layers having heights of $26-36 \AA$. The twice value of the particle height is almost the same with the long spacings estimated by out-of-plane XRD. The $\mathrm{PABA}_{7}$ and $\mathrm{PABA}_{8}$ monolayers show shapeless domains. In the case of the $\mathrm{PABA}_{17}$ monolayer, the formation of giant circular domains with a diameter of $20 \mathrm{~nm}$ is observed. Moreover, the surface of this domain is flat on a mesoscopic level.

Since the average height of these particles is 2.8 $\mathrm{nm}$, periodic structure of double particle layers is corresponding to result of out-of plane XRD.
Hydrophilic groups such as carbonyl groups are formed in these polymer particles at the air/water interface, and these groups are assumed to be localized at the bottoms of particles. Therefore, these particles do not have a completely homogenous structure, and it is considered that the spacing between the localized hydrophilic regions in the double particle layer can be estimated by performing out-of plane XRD measurements. In mesoscopic-scale images of the $\mathrm{PABA}_{7}$ monolayer, the presence of a sinewy domain is confirmed. According to the in-plane and out-of plane XRD measurement results, the molecular arrangement in these shapeless domains is almost random. $\mathrm{PABA}_{17}$ molecules are closely packed, and they form a side chain hexagonal lattice in a giant flat domain of monolayer surface. This hypothesis is supported by both the in-plane and out-of plane XRD measurement and AFM observation results. The polydispersity of the domain is significant in a large scan area of $50 \times 50 \mu^{2}$. The PABA 17 monolayer is an assembly of circular domains of different sizes.

Figure 5 shows a schematic illustration of the aromatic polyamide films with methyl to pentyl groups $\left(\mathrm{PABA}_{1-5}\right)$, heptyl to octyl groups $\left(\mathrm{PABA}_{7-8}\right)$, and stearyl groups $\left(\mathrm{PABA}_{17}\right)$ as the side chain in the monolayers. The organized molecular films of $\mathrm{PABA}_{1-5}$ are found to be multiparticle layers, consisting of single particle polyamide layers. The hydrophilic groups in the individual particles are assumed to be localized at the particle bottoms. The surface morphology of the $\mathrm{PABA}_{7}$ and $\mathrm{PABA}_{8}$ monolayers shows a shapeless sinewy domain. On the other hand, the $\mathrm{PABA}_{17}$ monolayer forms a giant domain consisting of a closely packed hexagonal subcell.

As mentioned above, the crystal structure, two-dimensional molecular arrangement, and surface morphology of aromatic polyamide monolayers depend on the side chain lengths. In

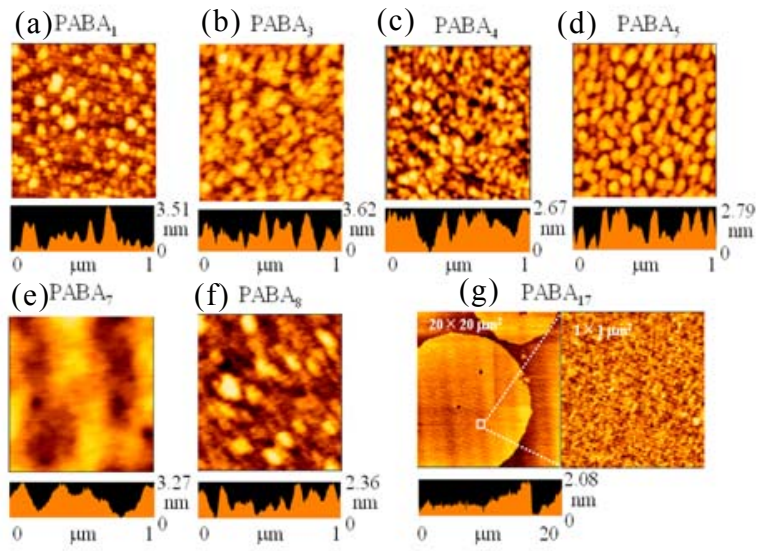

Figure 4 AFM images of Z-type monolayers on mica of aromatic polyamides with various side chains: (a) methyl, (b) propyl, (c) butyl, (d) pentyl, (e) heptyl, (f) octyl, and (g) stearyl substituents $\left(1 \times 1 \mu \mathrm{m}^{2}\right)$. 
(a) $\mathrm{PABA}_{1} \sim \mathrm{PABA}_{5}$ : Muliti-particle layer

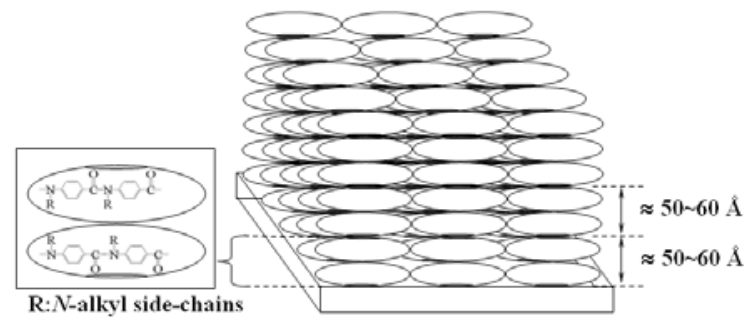

(b) $\mathrm{PABA}_{7}, \mathrm{PABA}_{8}$ Amorphous (Shapeless domain)

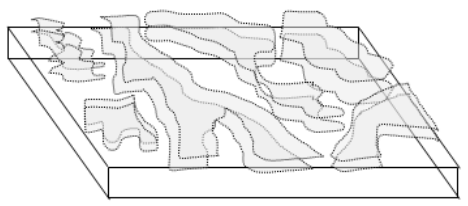

(c) $\mathbf{P A B A}_{17}$

Layer structure and sub-cell

formation in giant domain

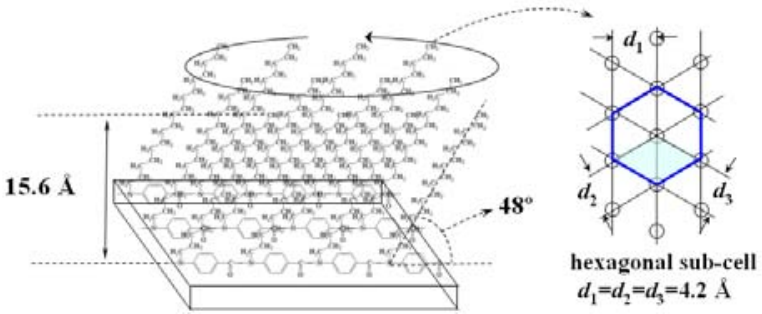

Figure 5 Schematic illustration of film structure (particle arrangement, mesoscopic morphology, or molecular packing) of (a) $\mathrm{PABA}_{1-5}$, (b) $\mathrm{PABA}_{7-8}$, and (c) $\mathrm{PABA}_{17}$.

other words, the method used to form organized molecular films of poly-( $N$-alkylated $)$ polyamides in this study can be directly used to control the molecular packing and formation of characteristic morphologies from subnanometer scale to mesoscopic scale. We believe that this study is the first to carry out a detailed structural analysis, particularly both molecular arrangement and morphology, of organized molecular films of poly-( $N$-alkylated polybenzamides $) \quad$ with systematic side chains.

\section{CONCLUSIONS}

The molecular arrangement and surface morphology, of organized molecular films of newly synthesized aromatic polyamides with different lengths were investigated by performing in-plane and out-of plane XRD measurements and carrying out AFM observations. The aromatic polyamide monolayers were highly condensed on the water surface at $15{ }^{\circ} \mathrm{C}$. The layered structure of $\mathrm{PABA}_{1}, \mathrm{PABA}_{3}, \mathrm{PABA}_{4}$, and $\mathrm{PABA}_{5}$ multilayers showed large spacings of $50-60 \AA$, as estimated from out-of plane XRD measurement results. The AFM observation results suggested that these aromatic polyamides formed single particle layers, wherein hydrophilic groups are localized at the bottom of the particles. The $\mathrm{PABA}_{7}$ and $\mathrm{PABA}_{8}$ multilayers showed irregularity and exhibited shapeless morphologies On the other hand, the organized molecular film of $\mathrm{PABA}_{17}$ formed a highly ordered layer structure (periodicity of $30 \AA$ ), side chain crystal showing two-dimensional hexagonal packing, and giant domain (diameter of $20 \mathrm{~nm}$ ). From these experimental findings, it is concluded that the polymer synthesis method employed in this study can be directly used to control the crystal structure, molecular arrangement, and surface morphologies of polymer monolayers.

\section{References}

[1] G. L. Gaines, Jr., "Insoluble Monolayers at Liquid Gas Interfaces", Wiley, New York, (1966).

[2] H. Kuhn, D. Möbius, H. Bucher, Spectroscopy of Monolayer Assemblies, in "Physical Methods of Chemistry", Vol. 1, (A. Weissberger, B. W. Rossiter, Eds.), Part IIIB, pp. 577-702, Wiley, New York, (1972).

[3] G. G. Roberts, "Langmuir-Blodgett Films", Plenum Press, London, (1990). M. C. Petty, "Langmuir-Blodgett Films", Cambridge Univ. Press, New York, (1996).

[4] K. Fukuda, Y. Shibasaki, H. Nakahara, Thin Solid Films, 160, 43-52 (1998).

[5] A. Keller, Phil. Mag., 2, 1171-75 (1957).

[6] K. Inomata, Y. Sakamaki, T. Nose, S. Sasaki, Polym. $J .$, 28, 986-91 (1996).

[7] V. Vand, Acta Cryst., 4, 465-469 (1951).

[8] G. Baskar, S. Ramya, A. B. Mandal, Colloid Polym. Sci., 280, 886-891 (2002).

[9] (a) V. P. Shibaev, N. A. Platé, Adv. Polym. Sci., 60/61, 173-252 (1984). (b) N. A. Platé, V. P. Shibaev, J. Polym., Sci., Mocromol. Rev., 8, 117-162 (1974)

[10] Y. Shibasaki, Y. Abe, N. Sato, A. Fujimori, Y. Oishi, Polym. J., 50, 72-80 (2010)

[11] A. Fujimori, Y. Sugita, H. Nakahara, E. Ito, M. Hara, N. Matsuie, K. Kanai, Y. Ouchi, K. Seki, Chem. Phys. Lett., 387, 345-251 (2004).

[12] F. Feng, M. Mitsuishi, T. Miyashita, I. Okura, K. Asai, Y. Amao, Langmuir, 15, 8673-8677 (1999).

(Received December 17, 2009; Accepted April 5, 2010) 